\title{
Clinical and serological characteristics of systemic sclerosis: Experience of a tertiary care center in Pakistan
}

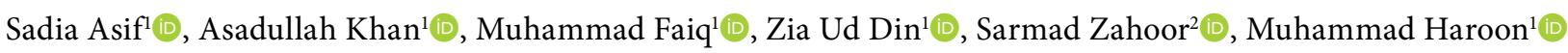 \\ ${ }^{1}$ Rheumatology, Fatima Memorial Hospital, Lahore, Pakistan \\ ${ }^{2}$ Department of Internal Medicine, King Edward Medical University, Mayo Hospital, Lahore, Pakistan
}

\begin{abstract}
Objectives: This study aims to evaluate the clinical and serological characteristics of systemic sclerosis (SSc) in Pakistani population.

Patients and methods: This prospective, cross-sectional study included a total of 38 patients (6 males, 32 females; mean age: $34.5 \pm 1.5$ years; range, 16 to 60 years) with SSc who were admitted to our rheumatology clinic between November 2019 and January 2020 . We evaluated the clinical, serological, and radiological features of SSc patients.

Results: Thirty-four (89.5\%) patients developed Raynaud phenomenon at the time of disease onset, while sclerodactyly was found in 34 (89.5\%), digital ulcers in 25 (65.8\%), and tendon friction rub in 12 (31.6\%) patients. Interstitial lung disease was present in 30 (78.9\%) patients with a higher prevalence in diffuse scleroderma (100\%) than in limited scleroderma $(70 \%)(p=0.01)$. Pulmonary hypertension was present in 18 patients with a significantly higher prevalence in diffuse disease $(57.1 \%)$ than limited disease $(11.8 \%)(p<0.01)$. Thirty $(78.9 \%)$ patients had impaired pulmonary function tests. Fibromyalgia was present in seven (18.4\%) patients, and depression was present in 10 (26.3\%) patients. Antinuclear antibody (ANA) was positive in 30 (78.9\%) patients. Anti-Scl-70 antibodies were present in $24(63.2 \%)$ patients with a significant association with diffuse disease $(85 \%$ vs. $35.3 \%$, respectively; $p<0.01)$. The anti-centromere antibodies (ACA) were present in $20(52.6 \%)$ patients with a significantly higher rate in limited disease $(94.2 \%$ vs. $19.0 \%$, respectively; $\mathrm{p}<0.01)$.
\end{abstract}

Conclusion: Scleroderma has a female preponderance. Raynaud phenomenon is the most initial clinical feature followed by other manifestations of a variable course and disease severity.

Keywords: Interstitial lung disease, Raynaud phenomenon, scleroderma, systemic sclerosis.

Systemic sclerosis (SSc) is an autoimmune disease of unknown etiology, characterized by fibrosis of the skin and internal organs of the body. This progressive, multisystem autoimmune disorder is characterized pathologically by vascular abnormalities, connective tissue sclerosis, and atrophy of the skin and various internal organs (e.g., alimentary tract, lungs, heart, kidney, blood vessels etc.). ${ }^{1}$ The prevalence of the disease varies among different populations worldwide.
The reported prevalence of SSc is 7.2 to 33.9 and 13.5 to 44.3 per 100,000 individuals in the Europe and North America, respectively. ${ }^{2}$

The disease involves various immunological, vascular, and inflammatory pathways leading to fibrosis of various organs. Skin and organ involvement in scleroderma are due to ongoing fibrosis and vasculopathy and superimposed thrombosis due to vessel injury. ${ }^{3}$ The diagnosis of SSc is mainly clinical, although certain criteria

Received: January 05, 2021 Accepted: April 26, 2021 Published online: October 24, 2021

Correspondence: Sarmad Zahoor, MD. Department of Internal Medicine, King Edward Medical University, Mayo Hospital, 54000 Lahore, Pakistan. Tel: +923336431557 e-mail: drsarmadzahoor@gmail.com

\section{Citation:}

Asif S, Khan A, Faiq M, Din ZU, Zahoor S, Haroon M. Clinical and serological characteristics of systemic sclerosis: Experience of a tertiary care center in Pakistan. Arch Rheumatol 2021;36(4):587-594. 
have been proposed to classify patients with scleroderma. The most commonly used criteria, known as the American College of Rheumatology (ACR)/European League Against Rheumatism (EULAR) classification criteria, were issued in 2013 as a collaborative effort. ${ }^{4}$ Clinically this disease can be divided into two main subtypes: (i) limited cutaneous SSc where there is skin involvement distal to elbows and knees and (ii) diffuse cutaneous SSc, with the involvement of skin of limbs extending proximal to elbows and knees along with the involvement of the trunk. ${ }^{5,6}$ Diffuse scleroderma manifests with multi-organ involvement such as interstitial lung disease (ILD), pulmonary hypertension, gastrointestinal (GI) involvement, and scleroderma renal crises. Early detection of such internal organ involvement in recent years has improved the long-term survival in diffuse scleroderma. ${ }^{7}$

Several scleroderma-specific autoantibodies have a prognostic importance in predicting specific organ involvement. Anti-topoisomerase I (anti-Scl-70) autoantibodies is associated with an increased risk of ILD, while the anti-ribonucleic acid (RNA) polymerase III autoantibodies are associated with scleroderma renal crisis and infrequently present in patients with lung disease. ${ }^{8}$ In this study, we aimed to evaluate the clinical and serological characteristics of SSc in Pakistani population.

\section{PATIENTS AND METHODS}

This prospective, descriptive, cross-sectional study was conducted at the Department of Rheumatology, Fatima Memorial Hospital, Lahore, Pakistan between November 2019 and January 2020. A total of 38 patients $(6$ males, 32 females; mean age: $34.5 \pm 1.5$ years; range, 16 to 60 years) who were newly and previously diagnosed with SSc and followed in our clinic were included in the study. Demographic data including age, sex, body mass index, socioeconomic and marital status, level of education; disease timelines such as the age of onset of illness, first symptom and interval between first symptom and rest of the disease manifestations, disease duration, initial most manifestations; clinical features such as constitutional, systemic manifestations, and detailed vascular features; and laboratory data including complete blood count $(\mathrm{CBC})$, renal functions and antibodies screening panels related to scleroderma such as ANA, ACA, anti-Scl-70, anti-RNA polymerase III antibody tests were collected for each patient. High-resolution computed tomography (HRCT) of the chest (reported by the consultant radiologist of the same institution reporting lung involvement as present, absent, and type of involvement as non-specific interstitial pneumonitis [NSIP] and usual interstitial pneumonitis [UIP]) were obtained for all patients. Echocardiography was performed to evaluate heart functions and as an indirect, non-invasive echocardiograph-based estimation of pulmonary hypertension (defined as a mean pulmonary arterial pressure of $\geq 25 \mathrm{mmHg}$ ). Pulmonary function tests including the estimation of lung volumes such as forced vital capacity (FVC), forced expiratory volume in $1 \mathrm{sec}$ (FEV1), and FEV1/FVC ratio were performed. Lung involvement was classified into mild (FEV1 $270 \%$ ), moderate (FEV1 50 to 69\%), and severe restriction (FEV1 $<50 \%)$. Diffusion lung capacity (DLCO) data were not included in the analysis due to missing data. Only the patients who fulfilled the ACR/EULAR diagnostic criteria for SSc were included. Patients aged below 16 years, those with a known history of lung dysfunction or chronic obstructive pulmonary disease, and those with scleroderma mimics such as morphea, scleroderma, eosinophilic fasciitis, or nephrogenic fibrosing dermopathy were excluded from the study. A written informed consent was obtained from each patient. The study protocol was approved by the Institutional Bioethics Review Committee (IBRC; No:FMH-092019-IRB-681-M). The study was conducted in accordance with the principles of the Declaration of Helsinki.

\section{Statistical analysis}

Statistical analysis was performed using the IBM SPSS version 25.0 software (IBM Corp., Armonk, NY, USA). All the categorical variables were presented in form of frequency and percentage whereas quantitative variables were presented in form of mean \pm standard deviation (SD). Comparison of categorical data between two groups was by chi-square test. For continuous data, Student's t-test was employed, along with the Mann-Whitney $U$ test when the data did not follow a normal distribution. Stratification 
of the data was done for type of scleroderma. Logistic regression model was applied for clinical associations using chi-square tests with $\mathrm{p}<0.05$ considered statistically significant.

\section{RESULTS}

Of the patients, those with diffuse scleroderma were significantly younger than those with limited scleroderma $(p=0.01)$. The female-to-male ratio was 16:3. Thirty-four (89.5\%) patients developed Raynaud phenomenon at the time of disease onset. The mean time from Raynaud phenomenon to other systemic features was $3.8 \pm 1.0$ years.
Demographic and clinical features of patients are summarized in Table 1.

Clinical manifestations of the overall cohort comprised of Raynaud phenomenon in all patients, sclerodactyly in 34 (89.5\%), digital ulcers in 25 (65.8\%), and tendon friction rubs in 12 (31.6\%) patients. Skin tightness proximal to elbow was present in nine (23.7\%) patients. Microstomia (decreased mouth opening) was present in 32 (84.2\%) patients. Twenty-eight (73.7\%) patients had arthritis with predominantly three patterns, polyarthritis being the most common. Respiratory symptoms included shortness of breath in 36 (94.7\%), palpitation in 24 (63.2\%), and chest

Table 1. Demographics and clinical features according to type of scleroderma

\begin{tabular}{|c|c|c|c|c|c|c|c|c|c|c|}
\hline \multirow[b]{2}{*}{ Variables } & \multicolumn{3}{|c|}{ Total $(n=38)$} & \multicolumn{3}{|c|}{ Limited ( $\mathrm{n}=17)$} & \multicolumn{3}{|c|}{ Diffuse $(n=21)$} & \multirow[b]{2}{*}{$p$} \\
\hline & $\mathrm{n}$ & $\%$ & Mean \pm SD & $\mathrm{n}$ & $\%$ & Mean \pm SD & $\mathrm{n}$ & $\%$ & Mean \pm SD & \\
\hline Age (year) & & & $34.5 \pm 1.5$ & & & $38.7 \pm 2.1$ & & & $31.1 \pm 1.9$ & 0.01 \\
\hline $\begin{array}{l}\text { Sex } \\
\quad \text { Male } \\
\text { Female }\end{array}$ & $\begin{array}{c}6 \\
32\end{array}$ & $\begin{array}{l}15.8 \\
84.2\end{array}$ & & $\begin{array}{c}1 \\
16\end{array}$ & $\begin{array}{l}16.7 \\
50.0\end{array}$ & & $\begin{array}{c}5 \\
16\end{array}$ & $\begin{array}{l}83.3 \\
50.0\end{array}$ & & 0.13 \\
\hline Age of diagnosis (year) & & & $30.2 \pm 1.6$ & & & $33.8 \pm 2.2$ & & & $27.0 \pm 2.1$ & 0.03 \\
\hline Raynaud at onset & 34 & 89.5 & & 15 & 88.2 & & 19 & 90.5 & & 1.00 \\
\hline Raynaud phenomenon & 38 & 100 & & 17 & 100 & & 21 & 100 & & - \\
\hline Sclerodactyly & 34 & 89.5 & & 17 & 100 & & 17 & 81 & & 0.11 \\
\hline Digital ulcers & 25 & 65.8 & & 11 & 64.7 & & 14 & 66.7 & & 1.00 \\
\hline Tendon friction rub & 12 & 31.6 & & 7 & 41.2 & & 5 & 23.8 & & 0.30 \\
\hline $\begin{array}{l}\text { Skin tightness } \\
\text { Distal to MCP } \\
\text { Distal to elbow } \\
\text { Proximal to elbow }\end{array}$ & $\begin{array}{c}23 \\
6 \\
9\end{array}$ & $\begin{array}{l}60.5 \\
15.8 \\
23.7\end{array}$ & & $\begin{array}{c}13 \\
2 \\
2\end{array}$ & $\begin{array}{l}76.5 \\
11.8 \\
11.8\end{array}$ & & $\begin{array}{c}10 \\
4 \\
7\end{array}$ & $\begin{array}{l}47.6 \\
19.0 \\
33.3\end{array}$ & & 0.17 \\
\hline Decreased mouth opening & 32 & 84.2 & & 17 & 100 & & 15 & 71.4 & & 0.02 \\
\hline Salt pepper appearance & 11 & 28.9 & & 7 & 41.2 & & 4 & 19 & & 0.16 \\
\hline Dry eyes and mouth & 7 & 18.4 & & 2 & 11.8 & & 5 & 23.5 & & 0.42 \\
\hline Oral ulcers & 12 & 31.6 & & 7 & 41.2 & & 5 & 23.8 & & 0.30 \\
\hline Arthritis & 28 & 73.7 & & 12 & 70.6 & & 16 & 76.2 & & 0.72 \\
\hline $\begin{array}{l}\text { Pattern of arthritis } \\
\text { Polyarthritis } \\
\text { Acro-osteolysis } \\
\text { Oligoarthritis }\end{array}$ & $\begin{array}{l}8 \\
2 \\
5\end{array}$ & $\begin{array}{l}53.3 \\
33.3 \\
33.3\end{array}$ & & $\begin{array}{l}4 \\
- \\
5\end{array}$ & $\begin{array}{c}44.4 \\
- \\
55.5\end{array}$ & & $\begin{array}{l}4 \\
2 \\
-\end{array}$ & $\begin{array}{c}66.7 \\
33.3 \\
-\end{array}$ & & 0.03 \\
\hline Shortness of breath & 36 & 94.7 & & 16 & 94.1 & & 20 & 95.2 & & 1.00 \\
\hline Palpitation & 24 & 63.2 & & 7 & 41.1 & & 18 & 85.7 & & 0.00 \\
\hline Chest pain & 7 & 18.4 & & 3 & 17.6 & & 4 & 19.0 & & 1.00 \\
\hline Regurgitation & 31 & 81.5 & & 12 & 70.5 & & 19 & 90.4 & & \\
\hline Dysphagia & 14 & 36.8 & & 5 & 29.4 & & 9 & 42.8 & & \\
\hline Primary infertility & 6 & 15.8 & & 4 & 23.5 & & 2 & 9.5 & & 0.05 \\
\hline Miscarriage & 14 & 36.8 & & 5 & 29.4 & & 9 & 42.9 & & 0.5 \\
\hline
\end{tabular}


Table 2. Baseline laboratory features of the cohort of scleroderma

\begin{tabular}{|c|c|c|c|c|c|c|c|c|c|c|}
\hline \multirow[b]{2}{*}{ Variables } & \multicolumn{3}{|c|}{ Total $(n=38)$} & \multicolumn{3}{|c|}{ Limited $(\mathrm{n}=17)$} & \multicolumn{3}{|c|}{ Diffuse $(n=21)$} & \multirow[b]{2}{*}{$p$} \\
\hline & $\mathrm{n}$ & $\%$ & Mean \pm SD & $\mathrm{n}$ & $\%$ & Mean \pm SD & $\mathrm{n}$ & $\%$ & Mean \pm SD & \\
\hline Antinuclear antibody & 30 & 78.9 & & 12 & 70.6 & & 18 & 85.7 & & 0.42 \\
\hline Anti-Scl-70 antibody & 24 & 63.2 & & 6 & 35.3 & & 18 & 85.7 & & 0.00 \\
\hline Anticentromere antibody & 20 & 52.6 & & 16 & 94.1 & & 4 & 19 & & 0.00 \\
\hline Hemoglobin & & & $12.2 \pm 0.2$ & & & $11.3 \pm 0.4$ & & & $12.9 \pm 0.3$ & 0.01 \\
\hline Total leukocyte counts & & & $9.6 \pm 0.7$ & & & $10.1 \pm 0.9$ & & & $9.3 \pm 1.0$ & 0.59 \\
\hline Platelets & & & $306.6 \pm 11.9$ & & & $315.2 \pm 20.5$ & & & $306.8 \pm 14.2$ & 0.72 \\
\hline Erythrocyte sedimentation rate & & & $48.2 \pm 4.9$ & & & $41.7 \pm 6.6$ & & & $53.5 \pm 7.0$ & 0.24 \\
\hline Creatinine & & & $0.8 \pm 0.0$ & & & $0.8 \pm 0.1$ & & & $0.7 \pm 0.0$ & 0.07 \\
\hline Alanine transaminase & & & $27.7 \pm 2.8$ & & & $35.4 \pm 5.6$ & & & $21.4 \pm 1.5$ & 0.01 \\
\hline
\end{tabular}

pain in seven (18.4\%) patients. Additionally, GI symptoms were regurgitation in 31 (81.5\%) and dysphagia in $14(36.8 \%)$ patients. Stratification of clinical features according to types of scleroderma showed a significantly younger age of onset $(p=0.03)$, microstomia $(p=0.02)$, and palpitation $(p=0.03)$ in diffuse scleroderma. Obstetric history showed a higher prevalence of primary infertility in at least six (15.8\%) patients with a significantly higher prevalence in limited SSc disease compared to diffuse disease $(23.5 \%$ vs. $9.5 \%$, respectively; $\mathrm{p}=0.05)$. Miscarriages occurring in $14(36.8 \%)$ patients were more common in diffuse disease than limited scleroderma $(42.9 \%$ vs. $29.4 \%$, respectively), although it did not reach statistical significance $(p=0.5)$.
Laboratory characteristics of the cohort including autoantibodies are shown in Table 2. The ANA was positive in 30 (78.9\%) patients. Anti-Scl-70 antibodies were present in 24 (63.2\%) patients with a significant association with diffuse disease $(85 \%$ vs. $35.3 \%$, respectively; $p<0.01)$, while ACAs were present in 20 (52.6\%) patients and significantly higher in limited disease $(94.2 \%$ vs. $19.0 \%$, respectively; $\mathrm{p}<0.01$ ).

Interstitial lung disease was present in $30(78.9 \%)$ patients with a higher prevalence in diffuse scleroderma (100\%) than in limited scleroderma (70\%) $(p=0.01)$. In addition, UIP was more common ILD pattern on HRCT in our cohort occurring in 17 (44.7\%) patients, followed

Table 3. Radiological investigations and pulmonary function tests according to type of disease

\begin{tabular}{|c|c|c|c|c|c|c|c|c|}
\hline & & $\mathrm{n}$ & $\%$ & $\mathrm{n}$ & $\%$ & $\mathrm{n}$ & $\%$ & $p$ \\
\hline \multicolumn{2}{|l|}{ Interstitial lung disease } & 30 & 78.9 & 9 & 52.9 & 21 & 100 & 0.01 \\
\hline \multirow{3}{*}{ HRCT pattern } & Normal & 8 & 21.0 & 7 & 41.1 & 7 & 41.1 & \multirow{3}{*}{0.03} \\
\hline & NSIP & 13 & 34.2 & 4 & 23.5 & 4 & 23.5 & \\
\hline & USIP & 17 & 44.7 & 6 & 35.3 & 6 & 35.3 & \\
\hline \multirow{4}{*}{ Pulmonary function tests } & Normal & 8 & 21.0 & 8 & 47.0 & - & - & \multirow[t]{4}{*}{0.001} \\
\hline & Mild & 5 & 13.1 & 3 & 17.6 & 2 & 9.5 & \\
\hline & Moderate & 11 & 28.9 & 2 & 11.8 & 9 & 42.8 & \\
\hline & Severe & 14 & 36.8 & 4 & 23.5 & 10 & 47.6 & \\
\hline \multicolumn{2}{|l|}{ Pulmonary hypertension } & 16 & 42.1 & 4 & 11.8 & 12 & 57.1 & 0.006 \\
\hline
\end{tabular}




\begin{tabular}{|c|c|c|c|c|c|c|c|}
\hline \multirow[b]{2}{*}{ Characteristics } & \multicolumn{2}{|c|}{ Total } & \multicolumn{2}{|c|}{ Limited } & \multicolumn{2}{|c|}{ Diffuse } & \multirow[b]{2}{*}{$p$} \\
\hline & $\mathrm{n}$ & $\%$ & $\mathrm{n}$ & $\%$ & $\mathrm{n}$ & $\%$ & \\
\hline Fibromyalgia & 7 & 18.4 & 3 & 17.6 & 4 & 19.0 & 1.0 \\
\hline Depression & 10 & 26.3 & 4 & 23.5 & 6 & 28.6 & 1.0 \\
\hline
\end{tabular}

by NSIP pattern in 13 (34.2\%) patients. Both of these patterns were significantly more common in diffuse scleroderma than limited disease $(p=0.03)$.

Pulmonary hypertension (defined as a mean pulmonary arterial pressure of $\geq 25 \mathrm{mmHg}$ ) was present in 18 (47.3\%) patients with a significantly higher prevalence in diffuse disease (57.1\%) than limited disease (11.8\%) ( $p<0.01)$. Thirty $(78.9 \%)$ patients were found to have impaired pulmonary function tests with a variable severity. Mild restrictive disease was present in five (13.1\%), moderate disease in 11 (28.9\%), and severe in 14 (36.8\%) patients. Severity was significantly associated with the type of disease, more severe restriction being manifested in diffuse disease $(\mathrm{p}<0.01)$ (Table 3).

Fibromyalgia diagnosed as per ACR criteria was present in seven (18.4\%) patients, and depression assessed by the Hospital Anxiety and depression (HADS) score was present in $10(26.3 \%)$ patients at the time of evaluation. There was no association of disease type with either of the two conditions $(p=1.0)$.

\section{DISCUSSION}

This study presents our experience of scleroderma at a tertiary care rheumatology center among Pakistani population. In Our study, female predominance, a higher percentage of diffuse type of scleroderma disease, and a higher prevalence of pulmonary hypertension were salient features.

The study has several important features to elaborate on. The female predominance with a female-to-male ratio of $16: 3$ is much lower than reported in regional cohorts such as Al-Adhadh and Al-Sayed ${ }^{9}$ (8.3:1) and Ghosh et al. ${ }^{10}$ (8.3:1). Cohorts from the other continents, however, have shown similar female predominance, as shown by
Carreira et al., ${ }^{11}$ Coi et al., ${ }^{12}$ Shanmugam et al., ${ }^{13}$ and Walker et al. ${ }^{14}$ from the European Scleroderma Trials and Research (EUSTAR) group. Besides, our cohort had a higher percentage of patients suffering from diffuse scleroderma, which is not consistent with the reported literature, showing a higher percentage suffering from limited disease. ${ }^{14}$ Such discrepancy can be attributed to geographical or ethnic variations. ${ }^{15}$ Besides, late referral and non-referral of cutaneous disease to the rheumatology center can be another reason, as most of these patients prefer dermatology consult than rheumatology due to the lack of systemic manifestations.

In the current study, there was a mean delay of $3.8 \pm 1.0$ years between Raynaud phenomenon and development of other systemic features. A cohort from Canada reported a delay of at least 6.2 years between Raynaud phenomenon and diagnosis of scleroderma in limited disease compared to diffuse disease. ${ }^{16}$ This important delay in manifestation of systemic features provide a crucial window of opportunity for early diagnosis of scleroderma before any organ involvement. A recent approach of the EUSTAR group called Very Early Diagnosis of Systemic Sclerosis (VEDOSS) proposed very early diagnosis in this window period by incorporating features such as Raynaud phenomenon, puffy hands, and capillary changes at nail folds with ANA serology. ${ }^{17}$ Such early diagnosis would ensure early treatment before any irreversible systemic manifestations.

In our study, none of the patients underwent right heart catheterization for estimation of pulmonary hypertension. However, non-invasive echocardiographic-based estimation of pulmonary hypertension was reported in $42.6 \%(n=18)$ of our cohort, with more prevalence in diffuse disease than limited scleroderma $(57.1 \%$ vs. $11.8 \%$, respectively). The findings are not consistent with the reported literature, both in prevalence and association with the type of scleroderma. 
The famous " $15 \%$ rule of scleroderma" states the prevalence of pulmonary hypertension around 15\%. ${ }^{18}$ The Early, Simple and Reliable Detection of Pulmonary Arterial Hypertension in Systemic Sclerosis (DETECT) study group, an international collaboration group, also reported a $19 \%$ prevalence of pulmonary hypertension in scleroderma. ${ }^{19}$ The non-invasive measurement of pulmonary hypertension using echocardiography alone can be an overestimation in our cohort and need to be confirmed either by gold-standard measurement such as right heart catheterization or compared with the algorithm proposed by the DETECT group. Besides, such a high prevalence in our cohort can be attributed to the fact that a higher number of our patients with diffuse disease had also ILD, which is not only a known cause of scleroderma-associated pulmonary hypertension, but also a poor prognostic factor. ${ }^{20}$

Arthritis in our study had a very high prevalence than previously reported literature. ${ }^{21,22}$ The reported prevalence of true synovitis (defined as joint tenderness and synovial swelling) ranges from $40 \%$ (Clement et al. ${ }^{23}$ ) to 60\% (Malcarne et al. ${ }^{24}$ ). However, the prevalence of joint involvement per se is much higher in scleroderma when detected on imaging techniques. Misra et al. ${ }^{25}$ reported a $91 \%$ prevalence of joint inflammation as detected by an imaging modality such as ultrasound. Arthralgias, myalgia, tenosynovitis, polyarthritis, and myositis are commonly reported musculoskeletal features. ${ }^{26}$ In our cohort, arthralgias followed by polyarticular type of arthritis were the predominant musculoskeletal features. Type of scleroderma did not show any association with arthritis in our study.

Depression and fibromyalgia are two main independent poor predictors of the disease. Depression in our cohort had a variable prevalence compared to other cohorts. March et al. ${ }^{27}$ reported a similar prevalence of $23 \%$ of depression. Other cohorts found a higher prevalence ranging from 46 to $56 \% .{ }^{28}$ Such diversity in prevalence may be due to using different screening tools in these studies. Similar to previous studies, depression was strongly associated with poor disease control, disease duration, and female sex. ${ }^{27,28}$

Similarly, the rate of fibromyalgia reported in our cohort was slightly lower than Perrot et al. ${ }^{29}$ who reported a prevalence of $(22 \%$ vs. $18 \%)$ and similar to Malcarne et al. ${ }^{30}$ Disease duration and activity are the predictors of fibromyalgia in scleroderma. ${ }^{31}$

The reproductive health of the cohort was another aspect of our study. Primary infertility was reported in $26 \%$ of patients and miscarriages in $50 \%$ of patients. The high percentage of primary infertility is not consistent with the reported literature. Several studies including Steen and Medsger ${ }^{32}$ and Giordano et al. $^{33}$ found no significant difference in infertility in scleroderma patients compared to controls. Miscarriages associated with scleroderma, however, was reported in previous studies, as reported by Giordano et al. ${ }^{33}$ and Ismail et al. ${ }^{34}$ Sobanski et al. ${ }^{35}$ also described severe organ involvement such as pulmonary hypertension, scleroderma renal crisis, and antiphospholipid antibody syndrome to be strongly associated with the increased fetal mortality.

Comparison with the international cohorts such as the Chinese arm of EUSTAR cohort, our study showed a similar prevalence of Raynaud phenomenon, cutaneous manifestations, and ILD; and a higher prevalence of digital ulcers (65\% vs. $28 \%)$, arthritis ( $73 \%$ vs. $27 \%)$, and pulmonary hypertension $(42 \%$ vs. $18 \%) .{ }^{36}$ The Genome Research in African American Scleroderma Patients (GRASP) cohort also showed similar demographic features of onset in young age (below 50 years), female predominance, and a higher percentage of diffuse disease (56\%). ${ }^{37}$ However, ILD was reported lower in that cohort (43\% vs. $78 \%)$.

Comparing with local and regional cohorts, our study showed consistent findings with the Khaliq et al.'s ${ }^{38}$ results in female predominance, age of onset of disease, clinical manifestations, and ANA positivity. However, the rate of diffuse scleroderma disease was much higher in our cohort compared to the aforementioned study (55\% vs. 38\%). Pradhan et al. $^{39}$ previously reported similar features of female predominance, higher percentage of diffuse systemic disease (58\% vs. $55 \%$ in our cohort) and a higher prevalence of ILD (88\% vs. $79 \%)$, pulmonary hypertension (62\% vs. $42 \%)$, and lower prevalence of Raynaud phenomenon (78\% vs. $100 \%)$, digital ulcers (23\% vs. $65 \%)$, and GI involvement ( $7.3 \%$ vs. $36 \%)$. 
Nonetheless, there are some limitations to this study. The study is a cross-sectional descriptive study and, therefore, the longitudinal characteristics were unable to be evaluated. In addition, being a single-center study, the results are difficult to generalize. Also, lung involvement was not sufficiently elaborated due to the lack of DLCO measurement as part of the pulmonary function test. Finally, pulmonary hypertension, estimated indirectly with echocardiography, was another limitation which might have biased the findings.

In conclusion, scleroderma in our population has a female predominance with a slightly higher percentage of diffuse type of disease. Besides, a crucial delay of diagnosis between Raynaud phenomenon and systemic disease provides an important window of early diagnosis. Interestingly, pulmonary hypertension is much more prevalent in our cohort probably secondary to the increased rate of ILD. Depression and fibromyalgia are also important comorbidities associated with disease duration and control.

\section{Declaration of conflicting interests}

The authors declared no conflicts of interest with respect to the authorship and/or publication of this article.

\section{Funding}

The authors received no financial support for the research and/or authorship of this article.

\section{REFERENCES}

1. Sobolewski P, Maślińska M, Wieczorek M, Łagun Z, Malewska A, Roszkiewicz M, et al. Systemic sclerosis - multidisciplinary disease: Clinical features and treatment. Reumatologia 2019;57:221-33.

2. Royle JG, Lanyon PC, Grainge MJ, Abhishek A, Pearce FA. The incidence, prevalence, and survival of systemic sclerosis in the UK Clinical Practice Research Datalink. Clin Rheumatol 2018;37:2103-11.

3. Yamamoto T. Scleroderma--pathophysiology. Eur J Dermatol 2009;19:14-24.

4. van den Hoogen F, Khanna D, Fransen J, Johnson SR, Baron M, Tyndall A, et al. 2013 classification criteria for systemic sclerosis: An American College of Rheumatology/European League against Rheumatism collaborative initiative. Arthritis Rheum 2013;65:2737-47.
5. Arkachaisri T, Fertig N, Pino S, Medsger TA Jr. Serum autoantibodies and their clinical associations in patients with childhood- and adult-onset linear scleroderma. A single-center study. J Rheumatol 2008;35:2439-44.

6. LeRoy EC, Medsger TA Jr. Criteria for the classification of early systemic sclerosis. J Rheumatol 2001;28:1573-6.

7. Nihtyanova SI, Tang EC, Coghlan JG, Wells AU, Black CM, Denton CP. Improved survival in systemic sclerosis is associated with better ascertainment of internal organ disease: A retrospective cohort study. QJM 2010;103:109-15.

8. Steen VD. Autoantibodies in systemic sclerosis. Semin Arthritis Rheum 2005;35:35-42.

9. Al-Adhadh RN, Al-Sayed TA. Clinical features of systemic sclerosis. Saudi Med J 2001;22:333-6.

10. Ghosh SK, Bandyopadhyay D, Saha I, Barua JK. Mucocutaneous and demographic features of systemic sclerosis: A profile of 46 patients from eastern India. Indian J Dermatol 2012;57:201-5.

11. Carreira PE, Carmona L, Joven BE, Loza E, Andréu $\mathrm{JL}$, Riemekasten G, et al. Differences associated with age at onset in early systemic sclerosis patients: A report from the EULAR Scleroderma Trials and Research Group (EUSTAR) database. Scand J Rheumatol 2019;48:42-51.

12. Coi A, Barsotti S, Santoro M, Almerigogna F, Bargagli E, Caproni $\mathrm{M}$, et al. Epidemiology of systemic sclerosis: A multi-database population-based study in Tuscany (Italy). Orphanet J Rare Dis 2021;16:90.

13. Shanmugam VK, Frech TM, Steen VD, Hummers LK, Shah AA, Bernstein EJ, et al. Collaborative National Quality and Efficacy Registry (CONQUER) for Scleroderma: Outcomes from a multicenter US-based systemic sclerosis registry. Clin Rheumatol 2020;39:93-102.

14. Walker UA, Tyndall A, Czirják L, Denton C, FargeBancel D, Kowal-Bielecka O, et al. Clinical risk assessment of organ manifestations in systemic sclerosis: A report from the EULAR Scleroderma Trials and Research group database. Ann Rheum Dis 2007;66:754-63.

15. Mayes MD, Lacey JV Jr, Beebe-Dimmer J, Gillespie BW, Cooper B, Laing TJ, et al. Prevalence, incidence, survival, and disease characteristics of systemic sclerosis in a large US population. Arthritis Rheum 2003;48:2246-55.

16. Hudson M, Thombs B, Baron M; Canadian Scleroderma Research Group. Time to diagnosis in systemic sclerosis: Is sex a factor? Arthritis Rheum 2009;61:274-8

17. Minier T, Guiducci S, Bellando-Randone S, Bruni $\mathrm{C}$, Lepri G, Czirják L, et al. Preliminary analysis of the very early diagnosis of systemic sclerosis (VEDOSS) EUSTAR multicentre study: Evidence for puffy fingers as a pivotal sign for suspicion of systemic sclerosis. Ann Rheum Dis 2014;73:2087-93. 
18. Muangchan C; Canadian Scleroderma Research Group, Baron M, Pope J. The 15\% rule in scleroderma: The frequency of severe organ complications in systemic sclerosis. A systematic review. J Rheumatol 2013;40:1545-56.

19. Coghlan JG, Denton CP, Grünig E, Bonderman D, Distler O, Khanna D, et al. Evidence-based detection of pulmonary arterial hypertension in systemic sclerosis: The DETECT study. Ann Rheum Dis 2014;73:1340-9.

20. Launay D, Mouthon L, Hachulla E, Pagnoux C, de Groote P, Remy-Jardin M, et al. Prevalence and characteristics of moderate to severe pulmonary hypertension in systemic sclerosis with and without interstitial lung disease. J Rheumatol 2007;34:1005-11.

21. Ostojic P, Damjanov N. Indices of the Scleroderma Assessment Questionnaire (SAQ) can be used to demonstrate change in patients with systemic sclerosis over time. Joint Bone Spine 2008;75:286-90.

22. Avouac J, Walker U, Tyndall A, Kahan A, Matucci-Cerinic M, Allanore Y; EUSTAR, et al. Characteristics of joint involvement and relationships with systemic inflammation in systemic sclerosis: Results from the EULAR Scleroderma Trial and Research Group (EUSTAR) database. J Rheumatol 2010;37:1488-501.

23. Clements PJ, Wong WK, Hurwitz EL, Furst DE, Mayes $\mathrm{M}$, White $\mathrm{B}$, et al. The Disability Index of the Health Assessment Questionnaire is a predictor and correlate of outcome in the high-dose versus lowdose penicillamine in systemic sclerosis trial. Arthritis Rheum 2001;44:653-61.

24. Malcarne VL, Hansdottir I, McKinney A, Upchurch $\mathrm{R}$, Greenbergs HL, Henstorf $\mathrm{GH}$, et al. Medical signs and symptoms associated with disability, pain, and psychosocial adjustment in systemic sclerosis. $J$ Rheumatol 2007;34:359-67.

25. Misra R, Darton K, Jewkes RF, Black CM, Maini RN. Arthritis in scleroderma. Br J Rheumatol 1995;34:831-7.

26. Morrisroe KB, Nikpour M, Proudman SM. Musculoskeletal manifestations of systemic sclerosis. Rheum Dis Clin North Am 2015;41:507-18.

27. March C, Huscher D, Preis E, Makowka A, Hoeppner $J$, Buttgereit $F$, et al. Prevalence, risk factors and assessment of depressive symptoms in patients with systemic sclerosis. Arch Rheumatol 2019;34:253-61.

28. Thombs BD, Taillefer SS, Hudson M, Baron M. Depression in patients with systemic sclerosis: A systematic review of the evidence. Arthritis Rheum 2007;57:1089-97.
29. Perrot S, Peixoto M, Dieude P, Hachulla E, Ottaviani $\mathrm{S}$, Allanore $\mathrm{Y}$. Incidence of Fibromyalgia Syndrome in Systemic Sclerosis and Rheumatoid Arthritis. Comparative Results According to Clinical Diagnosis, Screening Test with Fibromyalgia Rapid Screening Tool, Diagnosis with ACR1990 and ACR 2010 Criteria. The $76^{\text {th }}$ Annual Meeting of the ACR/ARHP, November 9-14 in Washington, USA: American College of Rheumatology; 2012. p. 1487.

30. Malcarne VL, Hansdottir I, McKinney A, Upchurch R, Greenbergs HL, Henstorf GH, et al. Medical signs and symptoms associated with disability, pain, and psychosocial adjustment in systemic sclerosis. J Rheumatol 2007;34:359-67.

31. Ayan A, Hekim H, Cetin SY. AB0547 Investigation of the presence of fibromyalgia syndrome in patients with scleroderma. Annals of the Rheumatic Diseases 2020;79:1570.

32. Steen VD, Medsger TA Jr. Fertility and pregnancy outcome in women with systemic sclerosis. Arthritis Rheum 1999;42:763-8.

33. Giordano M, Valentini G, Lupoli S, Giordano A. Pregnancy and systemic sclerosis. Arthritis Rheum 1985;28:237-8.

34. Ismail F, Abdel-Azeem MI, Abd El-Haleem $\mathrm{H}$, Mohammed AB, Katta MA, Mahmoud A, et al. Fetomaternal outcome in patients with systemic sclerosis. The Egyptian Rheumatologist 2013;35:101-6.

35. Sobanski V, Launay D, Depret S, Ducloy-Bouthors AS, Hachulla E. Special considerations in pregnant systemic sclerosis patients. Expert Rev Clin Immunol 2016;12:1161-73.

36. Hu S, Hou Y, Wang Q, Li M, Xu D, Zeng X. Prognostic profile of systemic sclerosis: Analysis of the clinical EUSTAR cohort in China. Arthritis Res Ther 2018;20:235.

37. Morgan ND, Shah AA, Mayes MD, Domsic RT, Medsger TA Jr, Steen VD, et al. Clinical and serological features of systemic sclerosis in a multicenter African American cohort: Analysis of the genome research in African American scleroderma patients clinical database. Medicine (Baltimore) 2017;96:e8980.

38. Khaliq T , Aziz W ,Farooqi A. Spectrum of systemic sclerosis in patients presenting to a tertiary care hospital in Pakistan. Pak Armed Forces Med J 2014;64:591-5

39. Pradhan V, Rajadhyaksha A, Nadkar M, Pandit P, Surve $\mathrm{P}$, Lecerf $\mathrm{M}$, et al. Clinical and autoimmune profile of scleroderma patients from Western India. Int J Rheumatol 2014;2014:983781. 